\title{
AVALIAÇ̃̃o DE CULTIVARES DE ALFACE ADUBADAS COM SILICATO DE CÁLCIO EM CASA-DE-VEGETAÇÃO ${ }^{1}$
}

\author{
Evaluation of lettuce cultivars fertilized with calcium silicate in greenhouse $^{1}$
}

\author{
Regina Lúcia Félix Ferreira ${ }^{2}$, Rovilson José de Souza ${ }^{3}$, Janice Guedes de Carvalho ${ }^{4}$, \\ Sebastião Elviro de Araújo Neto ${ }^{5}$, Vander Mendonça ${ }^{6}$, Paulo Guilherme Salvador Wadt ${ }^{7}$
}

\begin{abstract}
RESUMO
O experimento foi conduzido no Departamento de Ciência do Solo da Universidade Federal de Lavras - UFLA, no período de junho a agosto de 2002. Objetivou-se avaliar a produtividade, o estado nutricional e a qualidade (classe de tamanho) de cultivares de alface cultivadas com doses de silicato de cálcio em vasos sob casa-de-vegetação. O delineamento experimental foi em blocos casualizados, com quatro repetições, com 1 planta/vaso, em esquema fatorial 3 x $4+3$, sendo três cultivares de alface (Raider, Regina e Vera) e quatro doses de silicato de cálcio $\left(0,410,1,000\right.$ e 2,000 $\left.\mathrm{mg} \mathrm{dm}^{-3}\right)$; os tratamentos adicionais foram compostos pela aplicação de $820 \mathrm{mg} \mathrm{dm}^{-3} \mathrm{de}$ carbonato de cálcio, para as três cultivares. O estado nutricional das plantas de alface foi avaliado pelo Sistema Integrado de Diagnose e Recomendação (DRIS). A aplicação da fonte silicato de cálcio não aumentou o crescimento das plantas e não aumentou o teor dos nutrientes nas plantas de alface, mas melhorou a nutrição das plantas para Si e aumentou a porcentagem de plantas sadias. A aplicação também aumentou a concentração de Mn, devido ao alto conteúdo de Mn no fertilizante aplicado (Silifértil $\left.{ }^{\circledR}\right)$. As três variedades de alface comportaram-se como plantas não acumuladoras de Si.
\end{abstract}

Termos para indexação: Lactuca sativa L., nutrição mineral, resíduo siderúrgico.

\section{ABSTRACT}

The experiment was carried out at the Department of Soil Sciences of the Universidade Federal de Lavras - UFLA, from June to August 2002, with the objective to evaluate the productivity, the nutritional state and the quality (size class) of lettuce cultivars grown with calcium silicate in greenhouse. The experimental design was disposed in blocks with four replicates, in factorial arrangement with additional treatments: 3 x $4+3$, composed by three lettuce cultivars: Raider (group crisphead lettuce); Regina (group butterhead lettuce) and Vera (group looseleaf lettuce) and four calcium silicate rates $\left(0,410,1.000\right.$ and $\left.2.000 \mathrm{mg} \mathrm{dm}^{-3}\right)$, additional treatments were composed of the application of calcium carbonate of $1.000 \mathrm{mg} \mathrm{dm}^{-3}$ for the three cultivars. The nutritional status of lettuce was evaluated by Diagnosis and Integrated Recommendation System. The application of calcium silicate fertilizer did not increase the growth of the plants and did not improve the concentration of nutrients in lettuce plants; but improved the lettuce nutritional status for $\mathrm{Si}$ and increased the percentage of leaves healthy. The application also increase Mn concentration, due to high content of Mn in the silifértil ${ }^{\circledR}$ fertilizer. The three varieties of lettuce behaved as non-accumulation plants of Si.

Index terms: Lactuca sativa L., plant nutrition, siderurgical residue.

\section{(Recebido em 12 de setembro de 2008 e aprovado em 22 de maio de 2009)}

\section{INTRODUÇÃO}

A alface (Lactuca sativa L.) é uma espécie olerícola cultivada em todo território nacional, sensível às condições adversas de temperatura e umidade (Rodrigues et al., 1997), razão pela qual seu cultivo em casa-de-vegetação proporciona maior estabilidade de produção e maior precocidade (Bonnecarrère et al., 2000).

Apesar de considerado não essencial às plantas, estudos apontam a importância do Si no desenvolvimento das plantas (Kordorfer, 2006), principalmente quando a espécie é acumuladora desse elemento, como as gramíneas em geral, podem ainda aumentar o rendimento ou favorecer vários processos fisiológicos e bioquímicos desejáveis para as plantas (Kordörfer \& Datnoff, 1995)

Em alface americana, a aplicação de solução de silicato de potássio via foliar na dose de $2,1 \mathrm{~kg} \mathrm{ha}^{-1} \mathrm{de} \mathrm{Si}$ possibilitou maior massa fresca comercial por planta. Enquanto a massa fresca total responde ao efeito quadrático com maior rendimento na dose de $1,7 \mathrm{~kg} \mathrm{ha}^{-1} \mathrm{de}$ Si (Resende et al., 2003).

${ }^{1}$ Trabalho realizado como parte das exigências para titulação de Doutorado/UFLA

${ }^{2}$ Universidade Federal do Acre/UFAC - Departamento de Ciências Agrárias/DCA - Centro de Ciências Biológicas e da Natureza - Br 364 - Km 04 Distrito Industrial - Cx. P. 500 - 69915-900 - Rio Branco, AC - reginalff@yahoo.com.br

${ }^{3}$ Universidade Federal de Lavras/UFLA - Departamento de Agricultura/DAG - Lavras, MG

${ }^{4}$ Universidade Federal de Lavras/UFLA - Departamento de Ciência do Solo/DCS - Lavras, MG

${ }^{5}$ Universidade Federal do Acre/UFAC - Departamento de Ciências Agrárias/DCA - Rio Branco, AC

${ }^{6}$ Universidade Federal Rural do Semi-Árido/UFERSA - Departamento de Ciências Vegetais/VEG - Mossoró, RN

${ }^{7}$ Empresa Brasileira de Pesquisa Agropecuária/Embrapa - Rio Branco, AC 
O Si pode estimular o crescimento e a produção vegetal por meio de várias ações indiretas, como a diminuição do autossombreamento, deixando as folhas mais eretas; o decréscimo na suscetibilidade ao acamamento; a maior rigidez estrutural aos tecidos; a proteção contra estresses abióticos, como redução da toxidez de Al, Mn, $\mathrm{Fe}$ e Na; a diminuição da incidência de patógenos, o aumento da proteção contra herbívoros, incluindo os insetos fitófagos (Epstein, 1994; Marschner, 1995).

$\mathrm{O}$ uso de silicato de cálcio em alface pode promover maior rendimento físico e uma melhor qualidade (poucas folhas senescentes, danificadas por insetos ou doentes), pelo efeito positivo da absorção de $\mathrm{Si}, \mathrm{Ca}, \mathrm{Mg}, \mathrm{K}$ e outros nutrientes presentes nesta escória de siderurgia.

Neste sentido, o objetivo deste trabalho foi de avaliar a influência do Si da fonte de silicato de cálcio no rendimento físico, estado nutricional e na qualidade (classe de tamanho) de cultivares de alface cultivadas em vasos sob casa-de-vegetação.

\section{MATERIAL E MÉTODOS}

O experimento foi conduzido em casa-de-vegetação no período de 3 de junho a 20 de agosto de 2002, no Departamento de Ciência do Solo da Universidade Federal de Lavras - UFLA, em vasos preenchidos com solo classificado como LATOSSOLO VERMELHO Distroférrico, textura argilosa (Empresa Brasileira de Pesquisa Agropecuária - Embrapa, 2006), ácido e com baixa saturação de bases trocáveis. As condições de temperatura e UR na época de condução do experimento, médias dos meses de junho, julho e agosto, foi de $18,9^{\circ} \mathrm{C}$ de temperatura média e $62,7 \%$ de U.R.

O delineamento experimental foi em blocos casualizados com quatro repetições, com 1 planta vaso $^{-1}$, em esquema fatorial $3 \times 4$, com 3 tratamentos adicionais, sendo: três cultivares de alface Raider (grupo crespa repolhuda); Regina (grupo solta lisa) e Vera (grupo solta crespa); e quatro doses de silicato de cálcio $(0,410,820$ e $1,640 \mathrm{mg} \mathrm{dm}^{-3}$, respectivamente $0,500,1000$ e $2000 \mathrm{mg} \mathrm{dm}^{-3}$ de Silifértil ${ }^{\circledR}$. Os tratamentos adicionais foram compostos pela aplicação de $1.000 \mathrm{mg} \mathrm{dm}^{-3}$ de carbonato de cálcio $\left(\mathrm{CaCO}_{3}\right)$, para as três cultivares.

O solo peneirado foi colocado em vasos com capacidade para $5 \mathrm{dm}^{3}$, aplicando-se em todos os tratamentos $300 \mathrm{mg} \mathrm{dm}^{-3}$ de $\mathrm{N} ; 200 \mathrm{mg} \mathrm{dm}^{-3}$ de P; $300 \mathrm{mg} \mathrm{dm}^{-3}$ de K; 0,5 $\mathrm{mg} \mathrm{dm}{ }^{-3}$ de $\mathrm{B} ; 1,5 \mathrm{mg} \mathrm{dm}^{-3}$ de $\mathrm{Cu} ; 5 \mathrm{mg} \mathrm{dm}^{-3}$ de Zn e 0,1 $\mathrm{mg} \mathrm{dm^{-3 }}$ de Mo (Malavolta, 1980). A incorporação dos nutrientes foi feita por meio de solução nutritiva, sendo que, para $\mathrm{N}$ e $\mathrm{K}$, foram parcelados em três vezes, aos 10, 20 e 30 dias após o transplantio das cultivares, com exceção do fósforo, que foi previamente incorporado ao solo, e dos demais nutrientes $\mathrm{B}, \mathrm{Cu}, \mathrm{Zn}$ e Mo que foram aplicados em uma única vez no dia do transplantio.

A fonte de silício utilizada foi o silicato de cálcio, na forma comercial de Silifértil ${ }^{\circledR}$ (escória de siderurgia) composto de: $\mathrm{SiO}_{2}-42$ dag $\mathrm{kg}^{-1}, \mathrm{Ca}-40$ dag kg-1 $\mathrm{Mg}$ - 9 dag $\mathrm{kg}^{-1}, \mathrm{Fe}-1,9$ dag kg-1$, \mathrm{P}-1 \mathrm{dag} \mathrm{kg}^{-1}, \mathrm{~K}-1$ dag kg-1 $\mathrm{Mn}-6$ dag $\mathrm{kg}^{-1}, \mathrm{~S}-1 \mathrm{dag} \mathrm{kg}^{-1}, \mathrm{Zn}-2000 \mathrm{mg} \mathrm{kg} \mathrm{kg}^{-1}, \mathrm{~B}-500 \mathrm{mg} \mathrm{kg}{ }^{-1}$, $\mathrm{Cu}-500 \mathrm{mg} \mathrm{kg}{ }^{-1}$, Mo - $150 \mathrm{mg} \mathrm{kg}{ }^{-1}$, Co - $150 \mathrm{mg} \mathrm{kg}^{-1}$. A escória de siderurgia é a junção do calcário, o minério de ferro e o carvão, submetidos a uma temperatura de $1900^{\circ} \mathrm{C}$, que promove a redução do ferro e a produção de compostos indesejáveis que não foram reduzidos.

As mudas, obtidas em bandejas de isopor com substrato comercial, foram transplantadas 30 dias após a semeadura. A irrigação foi realizada diariamente com a quantidade de água reposta baseada na diferença de peso dos vasos, mantendo a umidade em $60 \%$ da capacidade de campo, determinada pelo método de Monteiro \& Frighetto (2000).

As plantas foram colhidas quando apresentaram máximo desenvolvimento vegetativo, aos 78 dias após a semeadura. As características avaliadas foram: massa fresca comercial, número de folhas comerciáveis, massa fresca e seca da parte aérea, massa seca de raiz, porcentagem de massa fresca de folhas senescentes e doentes e teores foliares de macronutrientes $(\mathrm{N}, \mathrm{P}, \mathrm{K}, \mathrm{Ca}, \mathrm{Mg}, \mathrm{S})$ e de micronutrientes ( $\mathrm{Mn}, \mathrm{Fe}, \mathrm{Zn}, \mathrm{Cu}, \mathrm{B}$ ) e do $\mathrm{Si}$.

A porcentagem de massa fresca de folhas senescentes foi calculada pela multiplicação do quociente da massa das folhas que senesceram naturalmente, folhas com danos e sintomas causados por pragas e patógenos pela massa total da planta multiplicado por 100 .

A parte aérea das plantas, posteriormente à análise da massa seca, foi moída e as amostras submetidas à digestão nitroperclórica para determinação dos teores de macro e micronutrientes, exceto $\mathrm{Ne} \mathrm{B}$, cujas amostras foram submetidas à digestão via seca. As concentrações de $\mathrm{Ca}$ e $\mathrm{Mg}$ foram determinadas por espectofotometria de absorção atômica; o K por fotometria de chama; P e B por colorimetria; S por turbidimetria e o $\mathrm{N}$ foi determinado pelo método de Kjeldahl (Malavolta et al., 1997). Os teores de Si foram determinados pelo método colorimétrico do "azul-demolibdênio" proposto por Gallo \& Furlani (1978).

Para a avaliação do estado nutricional das plantas, utilizaram-se os índices DRIS. As normas DRIS foram obtidas de um conjunto de 40 amostras foliares de alface das variedades Vera, Regina e Raider. As normas foram calculadas das amostras do tercil de maior peso comercial das plantas cultivadas em condições de casa-devegetação e em condições de campo, sendo 20 plantas 
para cada uma das condições de cultivo. A seguir, os índices DRIS foram calculados segundo a fórmula de Jones (1981), incluindo-se no cálculo do índice DRIS de cada nutriente, todas as relações, na forma direta e indireta (Bataglia \& Santos, 1990; Dias et al., 2010). Para a interpretação dos índices DRIS, adotou-se o critério de considerar deficiente a planta com índice DRIS negativo e suficiente a planta com índice DRIS positivo.

\section{RESULTADOS E DISCUSSÃO}

Em análise de variância mostrou-se haver diferença de forma significativa para as cultivares nas características: massa fresca da parte aérea, massa fresca comercial, número de folhas, massa seca da parte aérea e massa seca de raiz, $\mathrm{N}$, $\mathrm{K}, \mathrm{Mg}, \mathrm{Zn}$ e $\mathrm{Cu}$ (Tabelas 1 e 2). A diferença encontrada entre as cultivares é decorrente das características genéticas de cada uma, principalmente por serem de grupos diferentes.

Jones \& Handreck (1967) dividiram as plantas em três grupos distintos de acordo com a concentração de silicato $\left(\mathrm{SiO}_{2}\right)$ encontrado na matéria seca: 1$)$ acumuladoras de silício, contendo mais de $1 \%$ de silício e com uma relação molar silício/cálcio maior que 1, arroz e cana-de-açúcar são exemplos típicos desse grupo de plantas; 2) não acumuladoras, com menos de $0,5 \%$ de silício, exemplo típico é o tomateiro, que acumula a maior parte do silício nas raízes; 3 ) intermediárias, com 0,5 a 1,0\% de silício ou mais de $1 \%$ de silício, porém, com uma taxa silício/cálcio menor que 1\% (Epstein, 1995; Ma et al., 2001).

No tomate cultivar Floradada, mesmo não sendo uma planta acumuladora de silício, a aplicação de 2,8 $\mathrm{tha}^{-1}$ de Silifértil ${ }^{\circledR}$ promoveu maior produção e qualidade do produto (Carvalho et al., 2002).

A aplicação de silicato de cálcio diminuiu a porcentagem de folhas senescentes e doentes (Tabela 1). Por outro lado, não foram observadas diferenças significativas para o efeito do silicato de cálcio nos teores foliares de $\mathrm{N}\left(2,99 \mathrm{dag} \mathrm{kg}^{-1}\right), \mathrm{P}\left(0,24 \mathrm{dag} \mathrm{kg}^{-1}\right), \mathrm{K}\left(6,94 \mathrm{dag} \mathrm{kg}^{-1}\right)$, $\mathrm{Ca}\left(1,20 \mathrm{dag} \mathrm{kg}^{-1}\right), \mathrm{Mg}\left(0,06 \mathrm{dag} \mathrm{kg}^{-1}\right), \mathrm{S}\left(0,25 \mathrm{dag} \mathrm{kg}^{-1}\right), \mathrm{Si}$ $\left(0,18\right.$ dag kg-1 $\left.^{-1}\right), \mathrm{Fe}\left(113,94 \mathrm{mg} \mathrm{kg}^{-1}\right), \mathrm{B}\left(394,79 \mathrm{mg} \mathrm{kg}^{-1}\right), \mathrm{Zn}$ $\left(50,40 \mathrm{mg} \mathrm{kg}^{-1}\right)$ e $\mathrm{Cu}\left(9,46 \mathrm{mg} \mathrm{kg}^{-1}\right)$.

Este fato provavelmente é decorrente da alface não ser uma planta acumuladora de $\mathrm{Si}$, nas quais seria esperada correlação positiva da acumulação do Si com outros nutrientes, como N, P, K, Ca e Mg (Kordorfer et al., 1999; Ferreira et al., 2009), e correlação negativa com Al, Fe e Mn (Epstein, 1994; Marschner, 1995), muito embora a correlação da absorção de $\mathrm{Si}$ com outros nutrientes dependa também da composição da fonte de $\mathrm{Si}$, como ocorre com os resíduos de indústria (Defelipo et al., 1992).
Em plantas acumuladoras de $\mathrm{Si}$, há interação positiva com o N; assim, aplicações de doses adequadas de $\mathrm{N}$ na presença de $\mathrm{Si}$ aumenta a produção de matéria seca, como em arroz (Ma \& Takahashi, 1990). Doses crescentes de silicato de cálcio foram acompanhadas, na média das cultivares, pelo aumento da deficiência de $\mathrm{N}$, que foi de $-0,2$ no tratamento sem Si para até -0,7 para o tratamento com a maior dose do silicato de cálcio. Assim, a ausência de resposta do silicato de cálcio e uma maior produção de matéria seca poderiam ser explicados pela disponibilidade de $\mathrm{N}$ em teores subótimos.

Houve efeito significativo para o fator cultivar nos teores de $\mathrm{N}, \mathrm{K}, \mathrm{Mg}, \mathrm{Zn}$ e $\mathrm{Cu}$. As cultivares diferiram significativamente no teor de nitrogênio com aplicação de carbonato de cálcio (Tabela 3), com a Raider apresentando menor teor que as demais (Tabela 4).

A aplicação de silicato de cálcio promoveu acúmulo de zinco na cultivar Regina quando comparada com as cultivares Vera e Raider, que não diferiram estatisticamente entre si (Tabela 4). Em alface cultivado em campo, observase que teores de zinco pouco responde em função do aumento das doses de silicato de cálcio $\left(1,2\right.$ e $\left.4 \mathrm{t} \mathrm{ha}^{-1}\right)$ (Ferreira et al., 2009).

A cultivar Raider acumulou mais $\mathrm{Mg}, \mathrm{K}$ e $\mathrm{Cu}$ quando comparada com a cultivar Vera, e essa não diferiu estatisticamente da cultivar Regina (Tabela 4).

Observou-se uma interação entre as doses de silicato de cálcio e as cultivares para o teor de ferro (Tabela 1). Apenas a cultivar Vera respondeu à aplicação do silicato de cálcio (Figura 1). No entanto, não foi possível determinar um modelo de regressão para determinar as doses de máxima e mínima absorção de ferro que aumentou de 88,92 $\mathrm{mg} \mathrm{kg}^{-1}$ com 0 de silicato de cálcio, para $101,73 \mathrm{mg} \mathrm{kg}^{-1} \mathrm{e}$ $161,34 \mathrm{mg} \mathrm{kg}^{-1}$ nas doses de 410 e $820 \mathrm{mg} \mathrm{dm}^{-3}$, respectivamente e diminuindo para $85,30 \mathrm{mg} \mathrm{kg}^{-1}$ na dose de $1640 \mathrm{mg} \mathrm{dm}^{-3}$ de silicato de cálcio.

Foram observadas diferenças significativas para o fator silicato de cálcio no teor de Mn nas cultivares de alface (Tabela 1). O perfil dos teores de Mn se ajustaram ao modelo quadrático com ponto de máxima de $280,5 \mathrm{mg} \mathrm{kg}^{-1}$ na dose de $975,6 \mathrm{mg} \mathrm{dm}^{-3}$ (Figura 2). Talvez a fonte utilizada de silicato de cálcio, na forma comercial de Silifértil ${ }^{\circledast}$ (escória de siderurgia) composto com $6 \%$ de manganês tenha contribuído para esse resultado. Muito embora com o aumento da dose de silicato de cálcio, tenha havido uma redução do Mn, fato explicado por Jones \& Handreck (1967) em que o Si nas plantas alivia a toxicidade de manganês e do ferro, não somente porque reduz a absorção desses elementos, mas também porque aumenta o nível de tolerância interna ao excesso de manganês nos tecidos. 


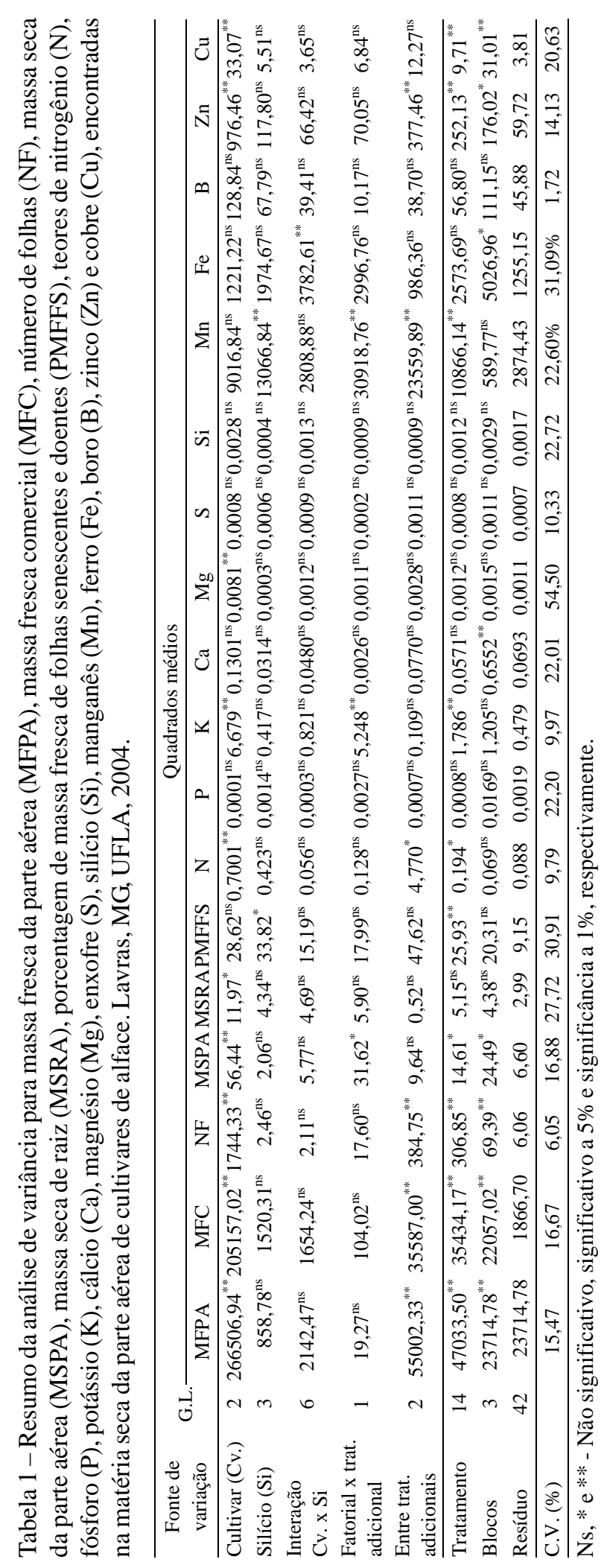

Ciênc. agrotec., Lavras, v. 34, n. 5, p. 1093-1101, set./out., 2010 
Tabela 2 - Efeito da aplicação de silicato de cálcio, em solo tipo Latossolo Vermelho Distroférrico sobre a massa fresca da parte aérea, massa fresca comercial, número de folhas, massa seca da parte aérea, massa seca de raiz e perda de massa. Lavras, MG, UFLA, 2004..$^{*}$

\begin{tabular}{|c|c|c|c|}
\hline \multirow{3}{*}{ Tratamentos } & \multicolumn{3}{|c|}{ Cultivares } \\
\hline & Raider & Regina & Vera \\
\hline & \multicolumn{3}{|c|}{ Massa fresca da parte aérea $(\mathrm{g})$} \\
\hline Silicato de cálcio $\quad 0 \mathrm{mg} \mathrm{dm}^{-3}$ & 414,25 & 211,25 & 235,00 \\
\hline Silicato de cálcio $410 \mathrm{mg} \mathrm{dm}^{-3}$ & 403,75 & 201,25 & 225,00 \\
\hline Silicato de cálcio $820 \mathrm{mg} \mathrm{dm}^{-3}$ & 446,00 & 194,75 & 231,25 \\
\hline Silicato de cálcio $1640 \mathrm{mg} \mathrm{dm}^{-3}$ & 479,25 & 189,00 & 222,25 \\
\hline Média & $435,81 \mathrm{a}$ & $199,06 \mathrm{~b}$ & $228,38 \mathrm{~b}$ \\
\hline \multirow[t]{2}{*}{$\mathrm{CaCO}_{3} 1000 \mathrm{mg} \mathrm{dm}^{-3}$} & $421,00 \mathrm{a}$ & $196,50 \mathrm{~b}$ & $250,50 \mathrm{~b}$ \\
\hline & \multicolumn{3}{|c|}{ Massa fresca comercial (g) } \\
\hline Silicato de cálcio $\quad 0 \mathrm{mg} \mathrm{dm}^{-3}$ & 374,75 & 190,00 & 213,33 \\
\hline Silicato de cálcio $410 \mathrm{mg} \mathrm{dm}^{-3}$ & 352,50 & 180,00 & 204,50 \\
\hline Silicato de cálcio $820 \mathrm{mg} \mathrm{dm}^{-3}$ & 400,33 & 180,25 & 202,50 \\
\hline Silicato de cálcio $1640 \mathrm{mg} \mathrm{dm}^{-3}$ & 431,75 & 181,00 & 206,75 \\
\hline Média & $389,91 \mathrm{a}$ & $182,81 \mathrm{~b}$ & $206,75 \mathrm{~b}$ \\
\hline \multirow[t]{2}{*}{$\mathrm{CaCO}_{3} 1000 \mathrm{mg} \mathrm{dm}^{-3}$} & $363,00 \mathrm{a}$ & $183,50 \mathrm{~b}$ & $223,00 \mathrm{~b}$ \\
\hline & \multicolumn{3}{|c|}{ Número de folhas } \\
\hline Silicato de cálcio $\quad 0 \mathrm{mg} \mathrm{dm}^{-3}$ & 26,75 & 43,25 & 21,75 \\
\hline Silicato de cálcio $410 \mathrm{mg} \mathrm{dm}^{-3}$ & 27,00 & 42,00 & 22,50 \\
\hline Silicato de cálcio $820 \mathrm{mg} \mathrm{dm}^{-3}$ & 26,75 & 42,75 & 23,00 \\
\hline Silicato de cálcio $1640 \mathrm{mg} \mathrm{dm}^{-3}$ & 27,25 & 72,75 & 24,50 \\
\hline Média & $26,94 \mathrm{~b}$ & $42,69 \mathrm{a}$ & $22,94 \mathrm{c}$ \\
\hline \multirow[t]{2}{*}{$\mathrm{CaCO}_{3} 1000 \mathrm{mg} \mathrm{dm}^{-3}$} & $25,00 \mathrm{~b}$ & $40,75 \mathrm{a}$ & $22,75 \mathrm{~b}$ \\
\hline & \multicolumn{3}{|c|}{ Massa seca da parte aérea $(\mathrm{g})$} \\
\hline Silicato de cálcio $\quad 0 \mathrm{mg} \mathrm{dm}^{-3}$ & 18,10 & 15,15 & 13,47 \\
\hline Silicato de cálcio $410 \mathrm{mg} \mathrm{dm}^{-3}$ & 17,24 & 14,73 & 15,24 \\
\hline Silicato de cálcio $820 \mathrm{mg} \mathrm{dm}^{-3}$ & 16,07 & 14,00 & 14,96 \\
\hline Silicato de cálcio $1640 \mathrm{mg} \mathrm{dm}^{-3}$ & 19,54 & 13,40 & 15,03 \\
\hline Média & $17,74 \mathrm{a}$ & $14,32 \mathrm{~b}$ & $14,68 \mathrm{~b}$ \\
\hline \multirow[t]{2}{*}{$\mathrm{CaCO}_{3} 1000 \mathrm{mg} \mathrm{dm}^{-3}$} & $14,99 \mathrm{a}$ & $12,02 \mathrm{a}$ & $14,29 \mathrm{a}$ \\
\hline & \multicolumn{3}{|c|}{ Massa seca da raiz (g) } \\
\hline Silicato de cálcio $\quad 0 \mathrm{mg} \mathrm{dm}^{-3}$ & 9,91 & 6,38 & 5,41 \\
\hline Silicato de cálcio $410 \mathrm{mg} \mathrm{dm}^{-3}$ & 6,45 & 5,84 & 6,62 \\
\hline Silicato de cálcio $820 \mathrm{mg} \mathrm{dm}^{-3}$ & 6,81 & 5,91 & 6,13 \\
\hline Silicato de cálcio $1640 \mathrm{mg} \mathrm{dm}^{-3}$ & 6,38 & 5,09 & 5,73 \\
\hline Média & $7,39 \mathrm{a}$ & $5,81 \mathrm{~b}$ & $6,04 \mathrm{ab}$ \\
\hline $\mathrm{CaCO}_{3} 1000 \mathrm{mg} \mathrm{dm}^{-3}$ & $5,21 \mathrm{a}$ & $5,91 \mathrm{a}$ & $5,73 \mathrm{a}$ \\
\hline
\end{tabular}


Tabela 2 - Continuação...

\begin{tabular}{|c|c|c|c|}
\hline \multicolumn{4}{|c|}{ Porcentagem de massa fresca de folhas senescentes e doentes $(\%)$} \\
\hline Silicato de cálcio $\quad 0 \mathrm{mg} \mathrm{dm}^{-3}$ & 13,47 & 10,16 & 9,23 \\
\hline Silicato de cálcio $410 \mathrm{mg} \mathrm{dm}^{-3}$ & 10,24 & 10,52 & 9,21 \\
\hline Silicato de cálcio $820 \mathrm{mg} \mathrm{dm}^{-3}$ & 9,72 & 7,66 & 12,46 \\
\hline Silicato de cálcio $1640 \mathrm{mg} \mathrm{dm}^{-3}$ & 10,01 & 4,37 & 7,15 \\
\hline Média & $10,86 \mathrm{a}$ & $8,18 \mathrm{~b}$ & $9,51 \mathrm{ab}$ \\
\hline $\mathrm{CaCO}_{3} 1000 \mathrm{mg} \mathrm{dm}^{-3}$ & $13,85 \mathrm{a}$ & $11,70 \mathrm{ab}$ & $7,10 \mathrm{~b}$ \\
\hline
\end{tabular}

*Médias seguidas de mesma letra na linha não diferem entre si, pelo teste de Tukey, a 5\% de probabilidade.

Tabela 3 - Indice DRIS para nitrogênio $(\mathrm{N})$, fósforo $(\mathrm{P})$, potássio $(\mathrm{K})$, cálcio $(\mathrm{Ca})$, magnésio $(\mathrm{Mg})$, silício $(\mathrm{Si})$, manganês $(\mathrm{Mn})$, ferro (Fe), boro (B) e cobre (Cu), encontradas na matéria seca da parte aérea de cultivares de alface. Lavras, MG, UFLA, 2004.

\begin{tabular}{|c|c|c|c|c|c|c|c|c|c|c|}
\hline Fonte de variação & $\mathrm{N}$ & $\mathrm{P}$ & $\mathrm{K}$ & $\mathrm{Mg}$ & $\mathrm{Ca}$ & $\mathrm{Si}$ & $\mathrm{Mn}$ & B & $\mathrm{Cu}$ & $\mathrm{Fe}$ \\
\hline \multicolumn{11}{|l|}{ Cultivar } \\
\hline Rider & $-0,20 \mathrm{a}$ & $-0,47 \mathrm{a}$ & $-0,11 \mathrm{a}$ & $0,15 \mathrm{a}$ & $0,37 \mathrm{c}$ & $-0,57 b$ & $0,53 \mathrm{a}$ & $-0,11 \mathrm{a}$ & $-0,28 b$ & $0,26 \mathrm{~b}$ \\
\hline Regina & $-0,92 b$ & $-0,48 \mathrm{a}$ & $-0,11 \mathrm{a}$ & $-0,12 b$ & $0,77 \mathrm{~b}$ & $0,25 \mathrm{a}$ & $0,25 \mathrm{~b}$ & $-0,74 b$ & $0,16 \mathrm{a}$ & $0,37 \mathrm{~b}$ \\
\hline Vera & $-0,24 \mathrm{a}$ & $-0,35 \mathrm{a}$ & $-0,02 \mathrm{a}$ & $0,10 \mathrm{a}$ & $1,08 \mathrm{a}$ & $-0,09 \mathrm{a}$ & $-0,77 \mathrm{c}$ & $-0,87 \mathrm{~b}$ & $-0,42 b$ & $0,75 \mathrm{a}$ \\
\hline \multicolumn{11}{|l|}{ Doses de Silifértil } \\
\hline Silicato de cálcio & $-0,20 \mathrm{a}$ & $-0,39 \mathrm{a}$ & $-0,11 \mathrm{a}$ & $-0,03 \mathrm{~b}$ & $0,84 \mathrm{a}$ & $-0,50 \mathrm{~b}$ & $-0,18 b$ & $-0,29 \mathrm{a}$ & $0,05 \mathrm{a}$ & $0,33 \mathrm{ab}$ \\
\hline Silicato de cálcio $410 \mathrm{mg} \mathrm{dm}^{-3}$ & $-0,36 a b$ & $-0,58 \mathrm{a}$ & $-0,15 \mathrm{a}$ & $0,002 \mathrm{ab}$ & $0,74 \mathrm{a}$ & $-0,17 \mathrm{ab}$ & $-0,08 \mathrm{~b}$ & $-1,07 b$ & $-0,35 \mathrm{a}$ & $0,74 \mathrm{a}$ \\
\hline Silicato de cálcio $820 \mathrm{mg} \mathrm{dm}^{-3}$ & $-0,52 \mathrm{ab}$ & $-0,52 \mathrm{a}$ & $0,04 \mathrm{a}$ & $0,14 \mathrm{a}$ & $0,75 \mathrm{a}$ & $0,25 \mathrm{a}$ & $0,00 \mathrm{~b}$ & $-0,01 \mathrm{a}$ & $-0,28 \mathrm{a}$ & $0,51 \mathrm{ab}$ \\
\hline Silicato de cálcio $1640 \mathrm{mg} \mathrm{dm}^{-3}$ & $-0,74 b$ & $-0,26 \mathrm{a}$ & $-0,11 \mathrm{a}$ & $0,06 \mathrm{ab}$ & $0,64 \mathrm{a}$ & $-0,13 \mathrm{ab}$ & $0,28 \mathrm{a}$ & $-0,93 \mathrm{~b}$ & $-0,14 \mathrm{a}$ & $0,25 \mathrm{~b}$ \\
\hline C.V. $(\%)$ & 71,3 & 61,6 & 412,0 & 476,0 & 64,1 & 435,0 & 174,67 & 415,00 & 420,00 & 84,1 \\
\hline
\end{tabular}

$\mathrm{Ns}, *$ e ** - Não significativo, significativo a $5 \%$ e $1 \%$ de probabilidade, respectivamente.

Tabela 4 - Teor de nitrogênio e potássio na matéria seca da parte aérea das três cultivares de alface cultivadas em vasos. Lavras, MG, UFLA, 2004.

\begin{tabular}{|c|c|c|c|c|c|c|}
\hline \multirow{3}{*}{ Cultivares } & \multirow{3}{*}{$\begin{array}{c}\text { Médias para o fator } \mathrm{CaCO}_{3} \\
\text { Nitrogênio } \\
\text { dag kg } \\
\end{array}$} & \multicolumn{5}{|c|}{ Médias para o fator Silicato de cálcio } \\
\hline & & Nitrogênio & Potássio & Magnésio & Zinco & Cobre \\
\hline & & \multicolumn{3}{|c|}{ dag $\mathrm{kg}^{-1}$} & \multicolumn{2}{|c|}{$\mathrm{mg} \mathrm{kg}^{-1}$} \\
\hline Raider & $2,82 \mathrm{~b}$ & $2,57 \mathrm{~b}$ & $7,50 \mathrm{a}$ & 0,083 a & $48,7 \mathrm{~b}$ & $10,8 \mathrm{a}$ \\
\hline Regina & $3,22 \mathrm{a}$ & $3,11 \mathrm{a}$ & $7,42 \mathrm{a}$ & 0,069ab & $58,2 \mathrm{a}$ & $9,2 \mathrm{ab}$ \\
\hline Vera & $3,14 \mathrm{a}$ & 3,16 a & $6,34 \mathrm{~b}$ & $0,039 \mathrm{~b}$ & 42,7 & $7,8 \mathrm{~b}$ \\
\hline C.V. & $9,79 \%$ & $9,79 \%$ & $8,37 \%$ & $54,5 \%$ & $15,3 \%$ & $25,6 \%$ \\
\hline
\end{tabular}

*Médias seguidas de mesma letra não diferem entre si, pelo teste de Tukey a 5\% de probabilidade.

Paralelamente, a aplicação de silicato de cálcio resultou em melhor equilibrio nutricional para o $\mathrm{Si}$, na média das cultivares, o qual foi de $-0,50$ no tratamento sem $\mathrm{Si}$ e de $-0,13$ a 0,24 para os tratamentos com as maiores doses de silicato de cálcio. Em relação às cultivares, a Raider foi a que apresentou a maior limitação por deficiência $(-1,65)$, e respondeu positivamente à aplicação de silicato de cálcio, independentemente da dose. A cultivar Vera também apresentou melhor equilibrio de Si com a aplicação de Silifértil ${ }^{\circledR}$, porém, em menor grau que a cultivar Raider. Por outro lado, a cultivar Regina aparentemente não respondeu à aplicação do silicato de cálcio. 


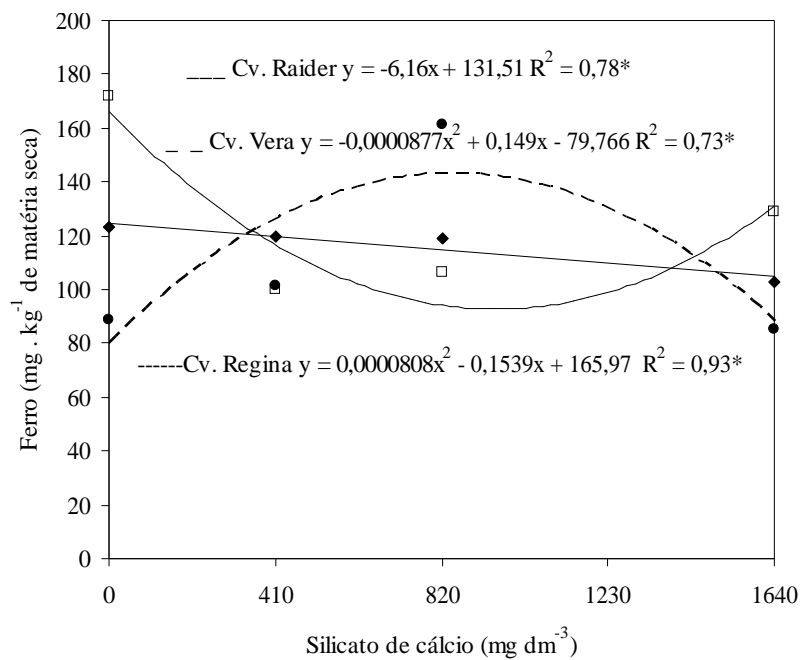

Figura 1 - Teores foliares de ferro $\left(\mathrm{mg} \mathrm{kg}^{-1}\right)$, na massa seca da parte aérea das três cultivares de alface cultivadas em vasos. Lavras, MG, UFLA, 2004.(1)

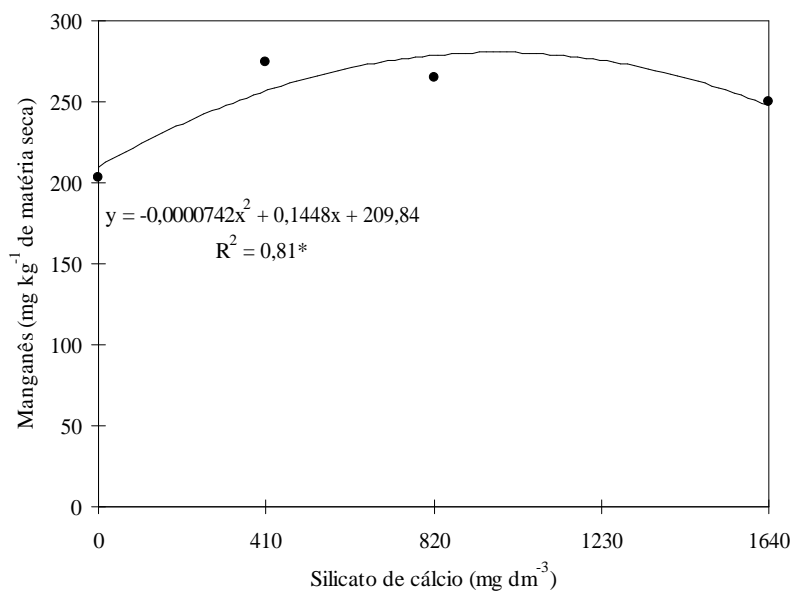

Figura 2 - Teores foliares de Mn na parte aérea das cultivares Raider, Regina e Vera. Valores provenientes da adubação com silicato de cálcio. Lavras, MG, UFLA, 2004.

Esses resultados indicaram que a cultivar Raider, em ambientes com maior disponibilidade de $\mathrm{Si}$, foi capaz de absorvêlo em proporções maiores que os demais nutrientes, até satisfazer um mínimo de exigência, a partir do qual, não mais respondeu à aplicação de maiores doses. Esse fenômeno é compatível com o comportamento esperado para cultivares que apresentam preferência pelo Si, como algumas americanas (Luz et al., 2006).

A diminuição da porcentagem de folhas senescentes e doentes (Figura 3) não pode ser explicada por um simples efeito na melhoria da acidez do solo, diminuindo teores de elementos tóxicos, como o Mn. Pelo fato do silicato de cálcio na forma de Silifértil ${ }^{\circledR}$ apresentar alto teor de $\mathrm{Mn}$, sua aplicação resultou em aumento nos teores de $\mathrm{Mn}$ das plantas, que foram acompanhadas também por maior absorção relativa de $\mathrm{Mn}$, em relação aos demais nutrientes.

O Si pode afetar a produção das plantas por várias ações indiretas, como melhorar a arquitetura das plantas, reduzir o acamamento, aumentando a rigidez estrutural dos tecidos, amenizando a toxidez por $\mathrm{Fe}, \mathrm{Mn}, \mathrm{Al}$ e $\mathrm{Na}$, e imprimir resistência às plantas ao ataque de pragas e doenças (Epstein, 1994; Marschner, 1995; Kordnorfer, 2006; Gomes et al., 2010). Nas plantas de alface, o efeito do silicato de cálcio pode ser explicado pela maior disponibilidade de Si nas plantas (indices DRIS tornando-se positivos), o que pode elevar os conteúdos de hemicelulose e lignina, aumentando assim a rigidez das células vegetais (Barbosa Filho et al., 2001). 


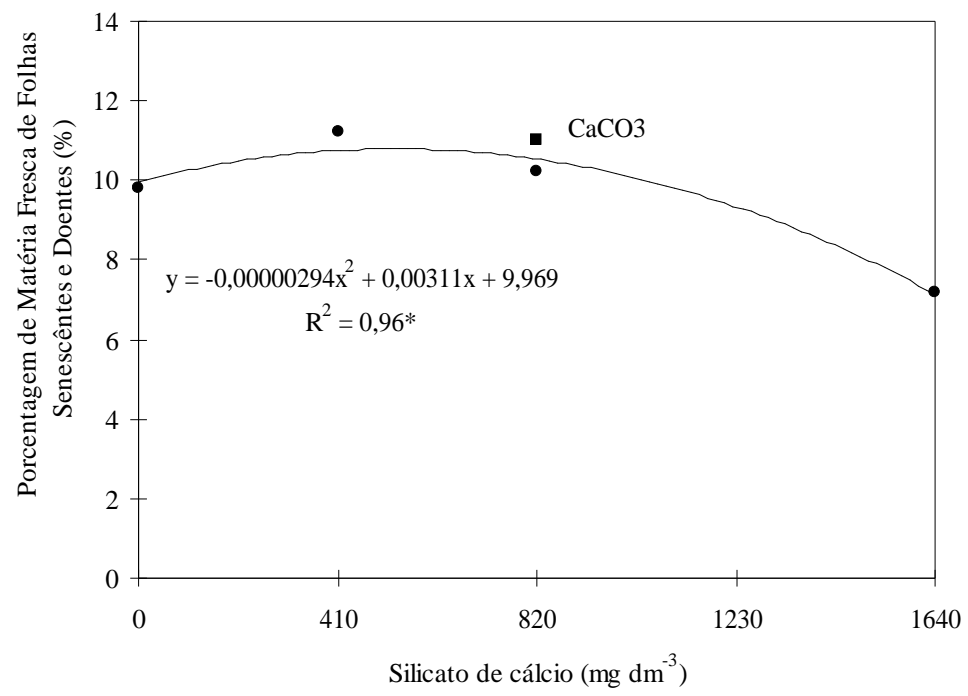

Figura 3 - Porcentagem de massa fresca de folhas senescentes e doentes de alface cultivada em vaso, com diferentes doses de silicato de cálcio. UFLA, Lavras - MG, 2004.

\section{CONCLUSÕES}

A aplicação de Si, na forma de silicato de cálcio, não promoveu aumento do crescimento das plantas cultivadas em vasos.

O aumento nas doses de silicato de cálcio proporcionou redução na porcentagem de massa fresca de folhas senescentes e doentes.

A aplicação de silicato de cálcio não alterou os teores da maioria dos nutrientes analisados nas cultivares de alface, com exceção dos teores de $\mathrm{N}$ e Mn.

As três cultivares de alface demonstraram ser plantas não acumuladoras de silício.

\section{REFERÊNCIAS BIBLIOGRÁFICAS}

BARBOSA FILHO, M.P.; SNYDER, G.H.; FAGERIA, N.K.; DATNOFF, L.E.; SILVA, O.F. Silicato de cálcio como fonte de silício para o arroz de sequeiro. Revista Brasileira de Ciências do Solo, Viçosa, v.25, p.325-330, mar./abr. 2001

BATAGLIA, O.C.; SANTOS, W.R. Efeito do procedimento de cálculo e da população de referência nos índices do Sistema Integrado de Diagnose e Recomendação (DRIS). Revista Brasileira de Ciências de Solo, Campinas, v.14, p.339-344, 1990.

BONNECARRÈRE, R.A.G.; LONDERO, F.A.A.; SANTOS, O.S. dos; SCHIMIDT, D. Desempenho de cultivares de alface em hidroponia no inverno.
Horticultura Brasileira, Brasília, v.18, n.2, p.289-291, 2000. Suplemento.

CARVALHO, J.G. de; MACHADO, A.Q.; NASCIMENTO, I.R. do; BOAS, R.C.V. Desempenho da cultura do tomate adubado com Silifértil. Horticultura Brasileira, Brasília, v.20, n.2, p.402, 2002. Suplemento 1.

DEFELIPO, P.V.; NOGUEIRA, A.V.; LOURES, E.G.; ALVAREZ, V.H. Eficiência agronômica de um resíduo de indústria siderúrgica. Revista Brasileira de Ciência do Solo, Campinas, v.16, n.1, p.127-131, jan./abr. 1992.

DIAS, J.R.M.; WADT, P.G.S.; LEMOS, C. de O.; DELARMELINDA, E.A.; SOLINO, J. da S.; TAVELLA, L.B.T. Relações nutricionais log-transformadas para avaliação nutricional de cupuaçueiros comerciais. Acta Amazônica, Manaus, v.40, n.1, p.37-42, Mar. 2010.

\section{EMPRESA BRASILEIRA DE PESQUISA} AGROPECUÁRIA. Sistema brasileiro de classificação de solos. Brasília, 1999. 412p.

EPSTEIN, E. The anomaly of silicon in plant biology.

Proceedings of National Academy of Sciences of the United States of America, Washington, v.91, n.1, p.11-17, Jan. 1994.

EPSTEIN, E. Photosynthesis, inorganic plant nutrition, solutions, and problems. Photosynthesis Nutrition, Dordrecht, v.15, n.1/2, p.37-39, Nov. 1995. 
FERREIRA, R.L.F.; SOUZA, R.J. de; CARVALHO, J.G. de; ARAÚJO NETO, S.E. de; YURI, J.E.Y. Avaliação de cultivares de alface adubadas com silifértil ${ }^{\circledR}$. Revista Caatinga, v.22, n.2, p.5-10, abr./jun. 2009.

GALLO, J.R.; FURLANI, P.R. Determinação de silício em material vegetal pelo método colorimétrico do azul de molibdênio. Bragantia, Campinas, v.37, n.2, p.5-11, 1978 .

GOMES, F.B.; MORAES, J.C.; NERI, D.K.P. Adubação com silício como fator de resistência a insetos-praga e promotor de produtividade em cultura de batata inglesa em sistema orgânico. Ciência e Agrotecnologia, Lavras, v.33, n.1, p.18-23, 2010.

JONES, C.A. Proposed modifications of the diagnosis and recommendation integrated system (DRIS) for interpreting plant analysis.

Communication Soil Science Plant Analisys, v.12, n.8, p.785-794, 1981.

JONES, L.H.P.; HANDRECK, K.A. Silica in soils, plants, and animals. Advances in Agronomy, New York, v.19, p.107-149, 1967.

MA, J.F.; MIYAKE, Y.; TAKAHASHI, E. Silicon as a beneficial element for crop plants. In: DATNOFF, L.E.; SNYDER, G.H.; KORNDORFER, G.H. (Eds.). Silicon in agriculture. New York: Elsevier Science, 2001. p.17-39.
MA, J.F.; TAKAHASHI, E. Effect of silicon on gorwth and phosphorus uptake of rice. Pant Soil, The Hague, v.126, p.115-119, 1990.

MALAVOLTA, E. Elementos de nutrição mineral de plantas. São Paulo: Agronômica Ceres, 1980. 251p.

MALAVOLTA, E.; VITTI, G.C.; OLIVEIRA, S.A. de. Avaliação do estado nutricional das plantas princípios e aplicações. 2.ed. Piracicaba: Potafós, 1997. 319p.

MARSCHNER, H. Mineral nutrition of higher plants. 2.ed. New York: Academic, 1995. 889p.

MONTEIRO, R.T.R.; FRIGHETTO, R.T.S. Capacidade de retenção de água do solo. In: FRIGHETTO, R.T.S.; VALARINE, P.S. (Coords.). Indicadores biológicos e bioquímicos da qualidade do solo. Jaguariúna: Embrapa Meio Ambiente, 2000. p.37-39.

RESENDE, M.G. de; YURI, J.E.; MOTA, J.H.; FREITAS, S.A.C. de; RODRIGUES JÚNIOR, J.C.; SOUZA, R.J. de; CARVALHO, J.G. de. Adubação foliar com silício em alface americana (Lactuca sativa $L$.) em cultivo de verão. Horticultura Brasileira, Brasília, v.21, n.2, p.374, 2003. Suplemento.

RODRIGUES, A.B.; MARTINS, M.I.E.G.; ARAÚJO, J.A.C. de. Avaliação econômica da produção de alface em estufa. Informações Econômicas, São Paulo, v.27, n.3, p.27-35, mar. 1997. 